\title{
Quantum for All
}

\author{
Propelling the development of quantum technologies will require \\ widespread literacy about quantum concepts, and a commitment to \\ diversity as a source of competitive advantage.
}

\author{
By Irfan Siddiqi, Darío Gil, and Joseph S. Broz
}

This piece is the second in a three-part series. Find out more about this series in this introductory article, Ingredients for a Quantum Future.

In the late 18th century, the English potter Josiah Wedgwood invented a clay pyrometer to measure the temperature of his kilns-declaring the ability to measure higher degrees of heat "an important acquisition, both to the philosopher and the practical artist" [1]. With his son, Thomas, he showed that different materials at the same temperature glow the same color. Combined with the optical prism, Wedgwood's thermal probe enabled newfound access to temperature and frequency information, giving humanity a new lease on light that ultimately challenged classical physics and led to the modern theory of quantum mechanics.

Today, new probes may once again allow humanity to make a technological leap-this time related to the bizarre notion that disparate and distant ions, photons, or other quantum objects can be highly correlated or "entangled." Thanks to a new

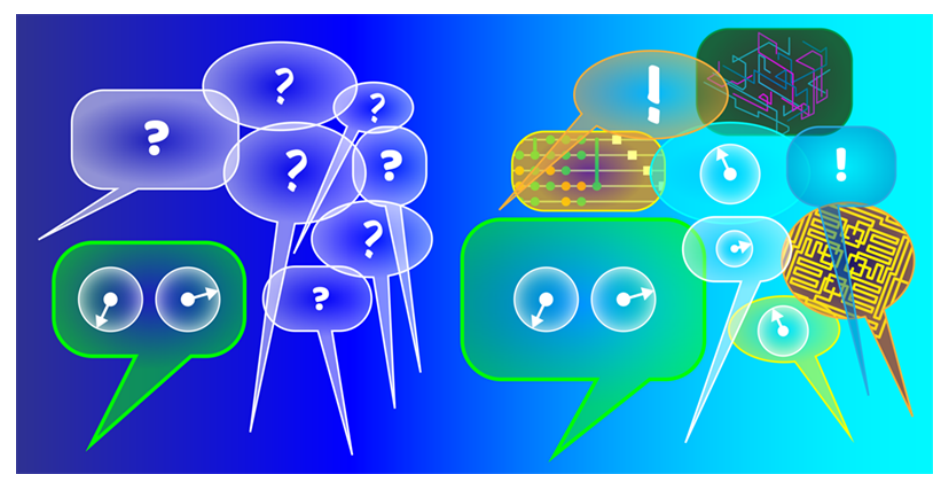

Credit: APS/Alan Stonebraker generation of tools to create, visualize, and manipulate quantum entanglement, we may soon see a revolution in sensing, communication, and computation. But if this technology is going to touch the lives of millions or more, then quantum mechanics must transition beyond a specialized physics course to a core requirement. Now is the time to consider the meaning of quantum literacy-specifically, what comprises a quantum canon and who needs to be versed in it?

Literacy is defined as being able to read and write, or as having a deep understanding and competency in a specific subject. By extension, quantum literacy has traditionally meant the ability to read, write, and understand the language of the physical world, as cast in the highly abstract, mathematical, and counterintuitive formalism of wave functions and operators. Mastery of this vernacular is an essential skill for hardware developers and computer science purists. But as the laws of quantum physics play a greater role in modern technology, R\&D managers, CEOs, and policy makers will need to become familiar with the major concepts of the theory-sans the mathematical acumen of being a card-carrying quantum mechanic. Quantum literacy thus needs to take on a broader meaning than proficiency in graduate-level physics.

How do we give the new inhabitants of the quantum ecosystem the right conceptual background? Traditional classes in quantum mechanics require a background in classical mechanics, electromagnetism, statistical mechanics, modern physics, differential equations, and linear algebra. Even after being equipped with this arsenal of education, one is faced with the challenge of having to apply the abstract equations that govern quantum wave functions to real-world systems. However, this abstraction needn't be an obstacle. Quantum mechanics is a masterfully ordered theory, with a precisely 
defined set of postulates and principles that predict how the world works in every situation that we have been able to test in the laboratory. Casting the formalism in the more common language of information theory would achieve a natural link between physics, computer science, materials science, and engineering. This interdisciplinary approach would form the core of an applications-focused academic curriculum that could be custom tailored for both purveyors and users of quantum technology.

Speaking the same language is a key step in propelling quantum technology into the next stages of development. It will require coordination between the different parts of the science and technology chain. For example, many quantum scientists are trained specialists who are asked to be a jack-of-all-trades because of their unique skills. These specialists often perform engineering tasks-setting up mechanical platforms, installing cryostats, or cajoling a laser system to produce an esoteric wavelength of light without flicker. As the industry matures, these activities will be transferred to suppliers and manufacturers, who will provide increasingly fit-for-use equipment to researchers and quantum hardware developers. Just as the digital developer today needs no understanding of semiconductor theory to do her job, the quantum worker will become increasingly divorced and segmented from the details of quantum physics. This step is a natural one toward efficiency and is the hallmark of a maturing industry. Such a transition will also open up the field, allowing a broader scientific community to engage with quantum technologies.

Critically, we have a unique opportunity to extend the spirit of openness in another dimension, which is in the makeup of the quantum workforce. Voices that emanate from a multicultural, multigenerational, and mixed-gender workforce will help drive innovation. A diverse team promotes the freedom to think out of the box. It also unleashes powerful forces-assuming good ideas can rise to the top irrespective of their origins. Science has historically been a common language that transcends borders and cultural differences. Incorporating inclusivity into the quantum ecosystem from the start will be essential for removing the geographical or cultural barriers that remain. Moreover, the history of much of American physics and engineering has been the history of the immigrant. The meritocracy of American universities will be squandered unless those building a quantum workforce vigorously pursue diversity in all its forms, including international cooperation.

Disclaimer: Dr. Siddiqi's views are his own and do not necessarily represent the views of the U.S. Department of Energy or the United States Government.

The next article in this series, Quantum at Scale, will look at the infrastructure needed to make quantum computing valuable to society at large.

Irfan Siddiqi: University of California, Berkeley, and Lawrence Berkeley National Laboratory, CA, USA

Darío Gil: IBM Research, Yorktown Heights, NY, USA

Joseph S. Broz: Quantum Economic Development Consortium (QED-C), Arlington, VA, USA

\section{REFERENCES}

1. J. Wedgwood, "An attempt to make a thermometer for measuring the higher degrees of heat, from a red heat up to the strongest that vessels made of clay can support," Phil. Trans. R. Soc. 72, 305 (1782). 